\title{
CROFTON MEASURES IN PROJECTIVE FINSLER SPACES
}

\author{
ROLF SCHNEIDER* \\ Mathematisches Institut, \\ Albert-Ludwigs-Universität, \\ Eckerstr. 1, D-79104, Freiburg i.Br., Germany \\ E-mail: rolf.schneider@math.uni-freiburg.de
}

\begin{abstract}
The classical Crofton formula of integral geometry expresses the area of a $k$ dimensional surface in Euclidean space as an integral, with respect to an invariant measure, of the number of intersection points with affine flats of the complementary dimension. This paper surveys attempts that have been made to obtain similar results in finite-dimensional normed spaces and in projective Finsler spaces. The stress is on relations to the theory of general (non-smooth) convex bodies and in particular to the geometry of zonoids.
\end{abstract}

The starting point of this introductory survey is a classical formula of integral geometry in Euclidean space. It interprets the volume of a submanifold as the measure of the set of flats of complementary dimension that hit the submanifold (counted with multiplicities). More precisely, let $M$ be a $k$-dimensional $C^{1}$ submanifold of Euclidean space $\mathbb{R}^{n}$, where $n \geq 2$ and $k \in\{1, \ldots, n-1\}$, and let $\lambda_{k}$ be the $k$-dimensional differential-geometric surface area measure. Let $A(n, j)$ denote the affine Grassmannian of $j$ flats ( $j$-dimensional affine subspaces) of $\mathbb{R}^{n}$. It carries an essentially unique Haar measure $\mu_{j}$ (a rigid motion invariant positive Borel measure which is finite on compact sets and not identically zero). An integral-geometric result known as the Crofton formula says that

$$
\int_{A(n, n-k)} \operatorname{card}(E \cap M) \mu_{n-k}(\mathrm{~d} E)=a_{n k} \lambda_{k}(M),
$$

where the constant $a_{n k}$ depends on the normalization of the measure $\mu_{n-k}$ (see, e.g., Santaló ${ }^{37}$, p. 245, (14.69)).

*Work partially supported by the European Network PHD, FP6 Marie Curie Actions, RTN, Contract MCRN-511953. 
In the following, we are interested in generalizations of (1) beyond Euclidean geometry and in reverse questions of the following kind. Suppose we are given a notion of area of $k$-dimensional surfaces that replaces $\lambda_{k}$; is it possible to represent it in the form (1)? In other words, we ask whether there exists a measure replacing $\mu_{n-k}$ in (1) so that the analogue of (1) holds for a large class of submanifolds $M$. By a measure on a locally compact space we understand in this survey a signed measure on the Borel sets of the space, which is finite on compact sets. A positive measure is a measure attaining only nonnegative values. A measure satisfying the generalized version of (1) as explained will be called a Crofton measure for the given notion of area. If such a formula exists, it connects metric notions, namely areas, with affine notions, namely flats; therefore, the existence can only be expected in situations where metric and affine structures are tied together in some way. A natural geometric environment of this kind is provided by the (general) projective Finsler spaces. Projective Finsler metrics are a special case of the projective metrics appearing in Hilbert's fourth problem. It is, in fact, the integral-geometric approach to Hilbert's fourth problem from which a natural development has led to the investigation of Crofton type formulas in projective Finsler spaces. We begin, therefore, our survey with a brief sketch of Hilbert's fourth problem and the role of integral geometry in its treatment.

\section{The Integral-geometric Approach to Hilbert's Fourth Problem}

The fourth problem in Hilbert's famous collection of 1900, entitled 'Problem von der Geraden als kürzester Verbindung zweier Punkte' (Problem of the straight line as the shortest connection of two points), was originally motivated by Hilbert's investigations into the foundations of geometry. Roughly speaking, it asks for the geometries, defined axiomatically, in which there exists a notion of length for which line segments are the shortest connections of their endpoints. The problem has later seen many transformations, generalizations as well as specializations, and we formulate here only a special case in later terminology:

H4. Given an open convex subset $C$ of $\mathbb{R}^{n}$, determine all complete projective metrics on $C$.

A metric $d$ on $C$ is called projective if it is continuous and satisfies

$$
d(p, q)+d(q, r)=d(p, r)
$$


whenever $p, q, r$ are points on a line, in this order. A metric satisfying (2) is also called linearly additive. For a given metric $d$, the length of a continuous parameterized curve $\gamma:[a, b] \rightarrow C$ is defined by

$$
L(\gamma):=\sup \sum_{i=1}^{k} d\left(\gamma\left(t_{i-1}\right), \gamma\left(t_{i}\right)\right),
$$

where the supremum is taken over all subdivisions $a=t_{0}<t_{1}<\cdots<$ $t_{k}=b, k \in \mathbb{N}$. For the segment $\overline{p q}$ with endpoints $p$ and $q$, (2) implies $L(\overline{p q})=d(p, q)$, and every continuous curve $\gamma$ with endpoints $p$ and $q$ satisfies $L(\gamma) \geq L(\overline{p q})$. Conversely, let $L$ be a notion of curve length for which $L(\overline{p q}) \leq L(\gamma)$ holds for every continuous curve from $p$ to $q$. It induces a metric $d$ by $d(p, q):=\inf L(\gamma)$, where the infimum is taken over all continuous curves from $p$ to $q$. This metric $d$ then satisfies $d(p, q)=L(\overline{p q})$ and, hence, also (2).

The notion of a projective metric is natural and fundamental: two basic structures, a metric and the linear structure of an affine space, are tied together by the compatibility condition (2). The determination of all projective metrics, however, is not an easy task. It is interesting to quote here from Busemann ${ }^{21}$ : “... Specifically, Hilbert asks for the construction of all these metrics and the study of the individual geometries. It is clear from Hilbert's comments that he was not aware of the immense number of these metrics, so that the second part of the problem is not a well posed question and has inevitably been replaced by the investigation of special, or special classes of, interesting geometries."

There are two classical examples of projective metrics, already given by Hilbert with the formulation of his fourth problem. The first example is that of a Minkowski space, that is, $\mathbb{R}^{n}$ with the metric induced by a norm $\|\cdot\|$. In that case, the distance defined by $d(x, y)=\|x-y\|$ is invariant under translations. The metrics coming from a norm are precisely the translation invariant projective metrics on $\mathbb{R}^{n}$. The second example is what is now called a Hilbert geometry. Here it is assumed that the open convex set $C$ is bounded. For $x, y \in C, x \neq y$, let $a, b$ be the points where the line through $x$ and $y$ meets the boundary of $C$, so that $a, x, y, b$ appear in this order on the line. With an auxiliary Euclidean norm $|\cdot|$, define

$$
d(x, y):=\ln \frac{|x-b||y-a|}{|y-b||x-a|} .
$$

Then $d$ is a projective metric on $C$.

Further examples of projective metrics do not easily come to mind. However, a wealth of them can be constructed by a nice integral-geometric 
approach suggested by Busemann, around 1960 (see Busemann ${ }^{20,19}$ ). Take any positive measure $\mu$ on the space $A(n, n-1)$ of hyperplanes of $\mathbb{R}^{n}$ which satisfies

$$
\mu(\{H \in A(n, n-1): p \in H\})=0 \quad \text { for each } p \in \mathbb{R}^{n}
$$

and

$$
0<\mu(\{H \in A(n, n-1): H \cap \overline{q p} \neq \emptyset\})<\infty \quad \text { for } p \neq q .
$$

If we define

$$
d(p, q):=\mu(\{H \in A(n, n-1): H \cap \overline{p q} \neq \emptyset\})
$$

for $p, q \in \mathbb{R}^{n}$, then $d$ is a projective metric. The triangle inequality $d(p, r) \leq$ $d(p, q)+d(q, r)$ follows from the fact that every hyperplane meeting the segment $\overline{p q}$ has to meet one of the segments $\overline{p q}$ or $\overline{q r}$. When $q$ lies in the segment $\overline{p r}$, then obviously (2) holds.

The question immediately arises whether this construction produces all projective metrics on $\mathbb{R}^{n}$. For the plane, the answer is affirmative. In independent work of Pogorelov ${ }^{36}$, Ambartzumian ${ }^{13}$, Alexander ${ }^{1}$, the following result was shown, in varying degrees of generality and with different formulations:

Theorem 1.1. The equation (6) establishes a linear isomorphism between the cone of projective metrics on $\mathbb{R}^{2}$ and the cone of positive measures $\mu$ on the space $A(2,1)$ of lines in $\mathbb{R}^{2}$ satisfying (4) and (5).

In dimensions greater than two, the situation is different; not every projective metric on $\mathbb{R}^{n}, n \geq 3$, can be obtained by Busemann's construction with a measure. This is already seen from the example of Minkowski spaces. The study of this case leads us in the next section to the zonoid equation, which plays an important role later on.

\section{The Zonoid Equation}

It is convenient in the following to use an auxiliary Euclidean structure on $\mathbb{R}^{n}$, given by a scalar product $\langle\cdot, \cdot\rangle$ with induced Euclidean norm $|\cdot|$. Then $S^{n-1}:=\left\{u \in \mathbb{R}^{n}:|u|=1\right\}$ is the Euclidean unit sphere. By $\sigma$ we denote the spherical Lebesgue measure on $S^{n-1}$. With the aid of the scalar product, we parameterize the hyperplanes $H \in A(n, n-1)$ in the form

$$
H_{u, t}=\left\{x \in \mathbb{R}^{n}:\langle x, u\rangle=t\right\}
$$

with $u \in S^{n-1}$ and $t \in \mathbb{R}$. 
Now let $\|\cdot\|$ be a norm on $\mathbb{R}^{n}$. Then

$$
B:=\left\{x \in \mathbb{R}^{n}:\|x\| \leq 1\right\}
$$

is the unit ball of the Minkowski space $\left(\mathbb{R}^{n},\|\cdot\|\right)$. We use the scalar product to identify $\mathbb{R}^{n}$ with its dual space, and hence identify the dual unit ball with the polar body of $B$,

$$
B^{o}:=\left\{u \in \mathbb{R}^{n}:\langle u, x\rangle \leq 1 \forall x \in B\right\} .
$$

Then

$$
h\left(B^{o}, \cdot\right)=\|\cdot\|
$$

is the support function of $B^{o}$.

Suppose now that the projective metric $d$ with $d(x, y)=\|x-y\|$ can be generated by (6), with a measure $\mu$ on $A(n, n-1)$ satisfying (4) and (5). It can be shown (Alexander ${ }^{2}$ ) that such a measure is unique. Since $d$ is translation invariant, the uniqueness implies that $\mu$ is translation invariant. We call the translation invariant measure $\mu$ on $A(n, n-1)$ satisfying

$$
\|x-y\|=\int_{A(n, n-1)} \operatorname{card}(H \cap \overline{x y}) \mu(\mathrm{d} H) \quad \text { for } x, y \in \mathbb{R}^{n}
$$

a Crofton measure for the norm $\|\cdot\|$. This is the simplest case of a Crofton measure for which there is a non-trivial existence problem; more general Crofton measures are the main topic of this survey.

The translation invariant (and, as generally assumed, locally finite) measure $\mu$ can be decomposed: there exists a finite measure $\varphi$ on the sphere $S^{n-1}$ such that

$$
\int_{A(n, n-1)} f \mathrm{~d} \mu=\int_{S^{n-1}} \int_{-\infty}^{\infty} f\left(H_{u, t}\right) \mathrm{d} t \varphi(\mathrm{d} u)
$$

holds for every nonnegative measurable function $f$ (see, for example, Schneider and Weil ${ }^{47}$, Satz 4.1.1, also for measures on $A(n, j)$ ). Since $H_{u, t}=H_{-u,-t}$, the measure $\varphi$ can be assumed to be even (i.e., to satisfy $\varphi(A)=\varphi(-A)$ for Borel sets $\left.A \subset S^{n-1}\right)$. Now the assumed representation $(6)$, or $(7)$, gives

$$
\|x\|=d(0, x)=\int_{S^{n-1}} \int_{-\infty}^{\infty} \mathbf{1}\left\{H_{u, t} \cap \overline{0 x} \neq \emptyset\right\} \mathrm{d} t \varphi(\mathrm{d} u),
$$

hence

$$
h\left(B^{o}, x\right)=\int_{S^{n-1}}|\langle u, x\rangle| \varphi(\mathrm{d} u) .
$$


The equation

$$
f(x)=\int_{S^{n-1}}|\langle u, x\rangle| \varphi(\mathrm{d} u), \quad x \in \mathbb{R}^{n},
$$

or specialized to

$$
f(x)=\int_{S^{n-1}}|\langle u, x\rangle| g(u) \sigma(\mathrm{d} u), \quad x \in \mathbb{R}^{n},
$$

where $f$ is a given even function and either the measure $\varphi$ or the measurable function $g$ is required, is known as the zonoid equation. This terminology has the following geometric background. Let $Z \subset \mathbb{R}^{n}$ be a convex body whose support function can be represented in the form

$$
h(Z, v)=\int_{S^{n-1}}|\langle u, v\rangle| \varphi(\mathrm{d} u), \quad v \in S^{n-1},
$$

with a positive even measure $\varphi$ on $S^{n-1}$. If $\varphi$ has finite support, then $h(Z, \cdot)$ is the sum of the support functions of finitely many (line) segments, hence $Z$ is the vector sum of finitely many segments. The convex bodies which are sums of finitely many segments are precisely the polytopes all of whose faces are centrally symmetric; they are called zonotopes, since their facets are arranged in zones. A convex body which can be approximated, in the Hausdorff metric, by zonotopes, is called a zonoid. Every zonoid has a centre of symmetry. A convex body with symmetry centre 0 is called centred. The convex bodies $Z$ representable by (12) with a positive measure $\varphi$ are precisely the centred zonoids. If $Z$, after a suitable translation, can be represented by (12) with a measure $\varphi$ (not necessarily positive), then it is called a generalized zonoid. If (12) holds with an even measure $\varphi$, then $\varphi$ is called the generating measure of $Z$, and a continuous density of $\varphi$ with respect to $\sigma$, if it exists, is called the generating function of $Z$.

If (10) is satisfied with an even measure $\varphi$, then we can use (8) to define a translation invariant measure $\mu$ on $A(n, n-1)$, and this satisfies (7).

About the zonoid equation, the following is known, for example. An even measure $\varphi$ satisfying (10) for given $f$ is uniquely determined. If the even function $f$ is sufficiently often differentiable (where 'sufficient' increases with the dimension), then (11) has a continuous solution $g$. This result goes back to Blaschke; a rigorous proof specifying differentiability assumptions was given in Schneider ${ }^{38}$; or see Schneider ${ }^{40}$, Th. 3.5.3. From this it follows that in the set of centrally symmetric convex bodies the generalized zonoids are dense, whereas the set of zonoids is closed and nowhere dense. Not every centrally symmetric convex body is a generalized zonoid. 
It follows from Schneider ${ }^{39}$, Lemma 6.1, that a convex body which has a face without a centre of symmetry cannot be a generalized zonoid.

For the existence of Crofton measures for norms, this has the following consequences.

Theorem 2.1. A Crofton measure for a norm exists if and only if the polar unit ball of the norm is a generalized zonoid.

For every sufficiently smooth norm on $\mathbb{R}^{n}$ there exists a Crofton measure, even one with a continuous density with respect to the motion invariant measure.

There are norms, for example that of $\ell_{\infty}^{n}$, for which no Crofton measure exists.

The existence of a positive Crofton measure is a much more restrictive property of a norm. To see this, choose $m$ points $p_{1}, \ldots, p_{m} \in \mathbb{R}^{n}$ and integers $N_{1}, \ldots, N_{m}$ with $\sum_{i=1}^{m} N_{i}=1$. Let $H$ be a hyperplane not incident with one of the points $p_{1}, \ldots, p_{m}$, and let $H^{+}, H^{-}$be the two closed halfspaces bounded by $H$. Then

$$
\begin{aligned}
& \sum_{i<j} 1\left\{\overline{p_{i} p_{j}} \cap H \neq \emptyset\right\} N_{i} N_{j} \\
& =\left(\sum_{p_{i} \in H^{+}} N_{i}\right)\left(\sum_{p_{j} \in H^{-}} N_{j}\right)=\left(\sum_{p_{i} \in H^{+}} N_{i}\right)\left(1-\sum_{p_{i} \in H^{+}} N_{i}\right) \leq 0 .
\end{aligned}
$$

If now (7) holds with a positive measure $\mu$, then integration over all hyperplanes with respect to $\mu$ gives

$$
\sum_{i<j} d\left(p_{i}, p_{j}\right) N_{i} N_{j} \leq 0 .
$$

A metric $d$ satisfying (13) for all $m$-tuples $\left(N_{1}, \ldots, N_{m}\right)$ of integers which sum to one, all $m \in \mathbb{N}$, and all $p_{1}, \ldots, p_{m} \in \mathbb{R}^{n}$, is called a hypermetric. We say that the Minkowski space $\left(\mathbb{R}^{n},\|\cdot\|\right)$ is hypermetric if its induced metric $d$ is a hypermetric.

We have shown: if $B^{o}$ is a zonoid, then $\left(\mathbb{R}^{n},\|\cdot\|\right)$ with $\|\cdot\|=h\left(B^{o}, \cdot\right)$ is hypermetric. The converse is also true, but lies deeper (references are in Schneider and Weil ${ }^{46}$, p. 301). We collect the results in the following theorem.

Theorem 2.2. For a norm $\|\cdot\|$ on $\mathbb{R}^{n}$ with unit ball $B$, the following conditions are equivalent: 
(a) There exists a positive Crofton measure for $\|\cdot\|$.

(b) The polar unit ball $B^{o}$ is a zonoid.

(c) The Minkowski space $\left(\mathbb{R}^{n},\|\cdot\|\right)$ is hypermetric.

The theorems of this section already indicate the role that zonoids and generalized zonoids play for the study of Crofton measures. This important role becomes even more evident in the generalizations considered in the sequel.

Surveys on zonoids were written by Bolker ${ }^{17}$, Schneider and Weil ${ }^{46}$, Goodey and Weil ${ }^{31}$. For background material on hypermetrics, we refer to Kelly $^{34}$ and to Deza and Laurent ${ }^{24}$.

\section{Projective Finsler Spaces}

Projective Finsler spaces are intermediate between Minkowski spaces and general projective metrics, in different respects. First, for a Finsler space, every tangent space is a Minkowski space. Second, sufficiently smooth projective metrics are induced from Finsler metrics, and projective metrics can be approximated, uniformly on compact sets, by smooth projective Finsler metrics.

In the following, we canonically identify the tangent space $T_{x} \mathbb{R}^{n}$ of $\mathbb{R}^{n}$ at the point $x$ with $\mathbb{R}^{n}$. Projective Finsler metrics can be defined on open convex subsets $C$ of $\mathbb{R}^{n}$. We define a (general) Finsler metric on $C$ as a continuous function $F: C \times \mathbb{R}^{n} \rightarrow[0, \infty)$ with the property that $F(x, \cdot)$ is a norm on $\mathbb{R}^{n}$, for each $x \in C$. Thus, we consider here only symmetric Finsler metrics, and we dispense with the differentiability assumptions common in Finsler geometry; this is a natural approach when we want to generalize arbitrary Minkowski spaces and Hilbert geometries. The length of a parameterized $C^{1}$ curve $\gamma:[a, b] \rightarrow C$ is defined by $\int_{a}^{b} F\left(\gamma(t), \gamma^{\prime}(t)\right) \mathrm{d} t$. The Finsler space $(C, F)$ is called projective if line segments are shortest curves connecting their endpoints; here it is not required that shortest connections are unique. The Finsler metric $F$ induces a metric $d_{F}$ by defining $d_{F}(p, q)$ as the infimum of the lengths of all piecewise $C^{1}$ curves connecting the points $p, q \in C$. If $(C, F)$ is projective, then the segment $\overline{p q}$ has length $d_{F}(p, q)$. If the metric $d_{F}$ is a hypermetric, then the Finsler space $(C, F)$ is called hypermetric.

We have already seen in Theorem 2.1 that, with regard to the existence of Crofton measures, there are essential differences between the smooth and non-smooth cases. For this reason, we have also to consider smooth Finsler 
metrics. The Finsler metric $F$ is said to be smooth if $F$ is of class $C^{\infty}$ on $C \times\left(\mathbb{R}^{n} \backslash\{0\}\right)$. (The further assumption common in Finsler geometry, namely that $F(x, \cdot)^{2}$ has positive definite Hessian on $\mathbb{R}^{n} \backslash\{0\}$, is not needed in the following.) If $F$ is a (smooth) Finsler metric on $C$, then $(C, F)$ is called a (smooth) Finsler space.

Let $(C, F)$ be a Finsler space. For each $x \in C,\|\cdot\|_{x}$ is a norm on $T_{x} \mathbb{R}^{n}=\mathbb{R}^{n}$, and the sets

$$
B_{x}:=\left\{\xi \in \mathbb{R}^{n}: F(x, \xi) \leq 1\right\}, \quad B_{x}^{o}:=\left\{u \in \mathbb{R}^{n}:\langle u, \xi\rangle \leq 1 \forall \xi \in B_{x}\right\}
$$

are, respectively, the unit ball and polar unit ball of this norm. The body $B_{x}$ is also called the indicatrix, and $B_{x}^{o}$ is called the figuratrix, of the Finsler metric $F$ at $x$. The role of the figuratrix in the calculus of variations is explained in Blaschke ${ }^{16}$.

If the Finsler space is hypermetric, then it can be shown (see Alexander ${ }^{2}$ ) that also the metric induced by $\|\cdot\|_{x}$ is a hypermetric, hence $B_{x}^{o}$ is a zonoid, for each $x \in C$.

Examples for projective Finsler spaces are, of course, the Minkowski spaces, but also the Hilbert geometries, since their metric is projective and is induced from a Finsler metric. This is seen as follows. Let $K \subset \mathbb{R}^{n}$ be a convex body with interior points and let $C$ be its interior. For distinct points $x, y \in C$, the Hilbert distance $d(x, y)$ is defined by (3); together with $d(x, x)=0$ this defines a projective metric $d$ on $C$ for which $(C, d)$ is complete. With $x, y, a, b$ as in (3), let $u \in \mathbb{R}^{n} \backslash\{0\}$ be a vector such that $b=x+t_{1} u, a=x-t_{2} u$ with $t_{1}, t_{2}>0$. For $\lambda>0$ with $x+\lambda u \in C$ we have

$$
d(x, x+\lambda u)=\ln \frac{t_{1}\left(t_{2}+\lambda\right)}{\left(t_{1}-\lambda\right) t_{2}}
$$

and hence

$$
F(x, u):=\lim _{\lambda \rightarrow 0} \frac{1}{\lambda} d(x, x+\lambda u)=\frac{1}{t_{1}}+\frac{1}{t_{2}} .
$$

This gives

$$
\begin{aligned}
\int_{0}^{1} F(x+\tau(y-x), y-x) \mathrm{d} \tau & =\int_{0}^{1}\left(\frac{1}{t_{1}-\tau}+\frac{1}{t_{2}+\tau}\right) \mathrm{d} \tau \\
& =\ln \frac{t_{1}\left(t_{2}+1\right)}{\left(t_{1}-1\right) t_{2}}=d(x, y) .
\end{aligned}
$$

Denoting by $\rho(M, \cdot)$ the radial function of a convex body $M$, we have

$$
t_{1}=\rho(K-x, u), \quad t_{2}=\rho(K-x,-u)
$$


and hence (using Schneider ${ }^{40}$, Remark 1.7.7)

$F(x, u)=h\left((K-x)^{o}, u\right)+h\left((K-x)^{o},-u\right)=h\left((K-x)^{o}-(K-x)^{o}, u\right)$.

We put $(K-x)^{o}=: K^{x}$ and have $K^{x}-K^{x}=\mathrm{D} K^{x}$, where $\mathrm{D}$ denotes the difference body operator, thus

$$
F(x, \cdot)=h\left(\mathrm{D} K^{x}, \cdot\right)
$$

(defining $F(x, 0):=0$ ). This shows that $F(x, \cdot)$ is a norm on $\mathbb{R}^{n}$, and its dual unit ball at $x \in C$ is given by

$$
B_{x}^{o}=\mathrm{D} K^{x} .
$$

Together with the observations above, this yields that $(C, F)$ is a projective Finsler space, and its induced metric $d_{F}$ is equal to $d$ (observe that $d_{F}(x, y)$ can also be obtained as the infimum of the Finsler lengths of all polygonal curves joining $x$ and $y$ ).

From now on we restrict ourselves, for simplicity of presentation, to Finsler metrics on $\mathbb{R}^{n}$.

We turn to Crofton measures. By a Crofton measure for the Finsler metric $F$ we understand a measure $\eta$ on $A(n, n-1)$ with the property that

$$
d_{F}(p, q)=\int_{A(n, n-1)} \operatorname{card}(H \cap \overline{p q}) \eta(\mathrm{d} H) \quad \text { for } p, q \in \mathbb{R}^{n} .
$$

We will now sketch how Pogorelov ${ }^{36}$ established the existence of Crofton measures for smooth projective Finsler metrics, using the zonoid equation.

Let $F$ be a smooth projective Finsler metric on $\mathbb{R}^{n}$. Since lines are extremals of the length integral, they must satisfy the Euler-Lagrange equations. From this, Pogorelov ${ }^{36}$, p. 63, deduces that

$$
\frac{\partial^{2} F(x, \xi)}{\partial x_{i} \partial \xi_{j}}=\frac{\partial^{2} F(x, \xi)}{\partial x_{j} \partial \xi_{i}}
$$

for $x=\left(x_{1}, \ldots, x_{n}\right), \xi=\left(\xi_{1}, \ldots, \xi_{n}\right) \neq 0$ and $i, j=1, \ldots, n$. (In a special case, this was already done by Hamel ${ }^{33}$. See Álvarez and Fernandes ${ }^{10}$ for a short proof of a generalization.) For every fixed $x \in \mathbb{R}^{n}$, Pogorelov now solves the zonoid equation for the function $F(x, \cdot)$. Since this function is of class $C^{\infty}$, there exists a continuous even function $\gamma(x, \cdot)$ on $S^{n-1}$ such that

$$
F(x, \xi)=\int_{S^{n-1}}|\langle\xi, u\rangle| \gamma(x, u) \sigma(\mathrm{d} u) \quad \text { for } \xi \in \mathbb{R}^{n} .
$$

Different from the approach in Pogorelov ${ }^{36}$, this can be done by using expansions in spherical harmonics, as in Schneider ${ }^{38}$ (see also Szabó ${ }^{49}$ ). With 
the aid of the estimates obtained in Schneider ${ }^{38}$, one can then show that the function $\gamma$ satisfies the differentiability assumptions, also with respect to the coordinates of $x$, to allow the following procedure. An argument of Pogorelov ${ }^{36}$, p. 63, deduces from (16) and (17) that

$$
\int_{S_{\xi}}\left(\frac{\partial \gamma(x, u)}{\partial x_{i}} u_{j}-\frac{\partial \gamma(x, u)}{\partial x_{j}} u_{i}\right) \sigma(\mathrm{d} u)=0
$$

holds for $i, j=1, \ldots, n$ and all $\xi \in S^{n-1}$, where $S_{\xi}:=\left\{u \in S^{n-1}:\langle\xi, u\rangle>\right.$ $0\}$. Since $\gamma(x, \cdot)$ is even, the integrand here is an odd function. An odd continuous function on $S^{n-1}$ with vanishing integrals over all hemispheres is identically zero (a result due to Funk ${ }^{27}$; see Schneider ${ }^{39}$, Korollar 3.2, for a proof of an extension, and Groemer ${ }^{32}$, Section 3.4, for a systematic treatment of the 'hemispherical transformation' and similar transformations by means of spherical harmonics). It results that

$$
\frac{\partial \gamma(x, u)}{\partial x_{i}} u_{j}-\frac{\partial \gamma(x, u)}{\partial x_{j}} u_{i}=0
$$

for $(x, u) \in \mathbb{R}^{n} \times S^{n-1}$. This means that, for fixed $u \in S^{n-1}$, the gradient of $\gamma(\cdot, u)$ is proportional to $u$, hence $\gamma(\cdot, u)$ is constant on every hyperplane with normal vector $u$. Therefore, we can define a continuous function $\Gamma$ : $A(n, n-1) \rightarrow \mathbb{R}$ by letting $\Gamma(H)$, for $H \in A(n, n-1)$, be the value that $\gamma(\cdot, u)$ attains on $H$, if $u$ is a unit normal vector of $H$ (it does not matter whether we choose $u$ or $-u$, since $\gamma(\cdot, u)=\gamma(\cdot,-u))$. Let $\eta$ be the measure on $A(n, n-1)$ which has the function $\Gamma$ as a density with respect to the rigid motion invariant measure (corresponding to the Euclidean structure) on $A(n, n-1)$, suitably normalized. We make this more explicit, and at the same time fix a normalization. Since $\gamma(\cdot, u)$ is constant on the hyperplane $H_{u, t}$, we can define a continuous function $g: S^{n-1} \times \mathbb{R} \rightarrow \mathbb{R}$ with $g(u, t)=$ $g(-u,-t)$ by means of $\gamma(x, u)=: g(u,\langle x, u\rangle)$. Then we can define the measure $\eta$ by

$$
\int_{A(n, n-1)} f \mathrm{~d} \eta:=\int_{S^{n-1}} \int_{-\infty}^{\infty} f\left(H_{u, t}\right) g(u, t) \mathrm{d} t \sigma(\mathrm{d} u)
$$

for every nonnegative measurable function $f$ on $A(n, n-1)$. The signed measure $\eta$ satisfies (15). This is shown by Pogorelov ${ }^{36}$ (for $n=2,3$ ), see also Szabó ${ }^{49}$, but will also follow from more general formulas below.

Since $F(x, \cdot)=\|\cdot\|_{x}$ is the support function of the polar unit ball $B_{x}^{o}$, we can write (17) in a form which will later be useful. So far, the following has been obtained. 
Theorem 3.1. If $\left(\mathbb{R}^{n}, F\right)$ is a smooth projective Finsler space, then there exists a uniquely determined continuous function $g: S^{n-1} \times \mathbb{R} \rightarrow \mathbb{R}$ with $g(u, t)=g(-u,-t)$ such that

$$
h\left(B_{x}^{o}, \xi\right)=\int_{S^{n-1}}|\langle\xi, u\rangle| g(u,\langle x, u\rangle) \sigma(\mathrm{d} u) \quad \text { for } x, \xi \in \mathbb{R}^{n} .
$$

Therefore, a Crofton measure exists for the metric $d_{F}$.

The equation (19) has, in particular, the following consequence. For $x, y \in \mathbb{R}^{n}$, the generating functions of $B_{x}^{o}$ and $B_{y}^{o}$ coincide on the subsphere $s_{x-y}$, where $s_{u}:=S^{n-1} \cap u^{\perp}$; here $u^{\perp}$ is the linear subspace through 0 orthogonal to $u$. Thus, in a smooth projective Finsler space, the unit balls at different points are strongly tied together and can never be chosen independently. Considerations on the construction of projective Finsler metrics are found in $\mathrm{Hamel}^{33}$ and Ambartzumian and Oganian ${ }^{14}$.

To obtain the above results, strong smoothness assumptions are necessary, though weaker differentiability assumptions than $C^{\infty}$ are sufficient. For general projective metrics, there are approximation results due to Pogorelov $^{36}$ (for $n=2,3$ ) and Szabó ${ }^{49}$. These authors have shown that projective metrics can be approximated suitably by smooth projective Finsler metrics. The corresponding sequences of Crofton measures will in general not contain vaguely convergent subsequences, so that the value of these approximations is limited. This is different, though, if the measures are positive. Therefore, further conclusions can be drawn in the case of projective hypermetrics. In that case, Alexander ${ }^{2}$ has shown that also the approximating Finsler spaces are hypermetric and that, therefore, all the local polar unit balls are zonoids. The corresponding Crofton measures are then positive measures, and Alexander ${ }^{2}$ was able to deduce the following result.

Theorem 3.2. The equation (6) establishes a linear isomorphism between the cone of projective hypermetrics on $\mathbb{R}^{n}$ and the cone of positive measures $\mu$ on the space $A(n, n-1)$ of hyperplanes in $\mathbb{R}^{n}$ satisfying (4) and (5).

This can be considered as a solution of Hilbert's fourth problem for the special case of projective hypermetrics. For smooth projective Finsler metrics, Pogorelov's and Szabó's results establish the existence of a Crofton measure. This is in general not positive, but has been called quasipositive, since it has the following property. If $\overline{x y}$ and $\overline{y z}$ are non-collinear segments, then the measure of the set of hyperplanes intersecting both segments is positive. An effective criterion for quasi-positive measures seems 
to be lacking, so that the construction does not yield a complete explicit description of the smooth projective Finsler metrics. The remaining question is essentially the same as the question for the conditions which a function $g$ in (19) has to satisfy so that the integral defines a support function. With these remarks, we leave Hilbert's fourth problem, since our main concern is the existence of Crofton measures for higher dimensional areas instead of lengths. We mention, however, that a thorough investigation of general projective metrics was undertaken by Szabó ${ }^{49}$, and that in the smooth case there is an elegant approach via symplectic geometry due to Álvarez ${ }^{5}$. Álvarez ${ }^{4}$ has also written a beautiful introduction, at an elementary level, to the planar case of Hilbert's fourth problem.

\section{Notions of Area}

Generalizing Hilbert's fourth problem, Busemann ${ }^{20,19}$ proposed to study axiomatically defined notions of $k$-dimensional areas in $n$-dimensional affine spaces for which $k$-flats minimize area. In close connection with this, he also suggested to study the $k$-dimensional areas that satisfy a Crofton formula. A different approach to Crofton type results are the analytic investigations of Gelfand and Smirnov ${ }^{29}$, Álvarez, Gelfand and Smirnov ${ }^{11}$ on Crofton $k$ densities, aiming at connecting the two classical integral geometries, that of Poincaré, Blaschke, Chern on the one hand, and the integral geometry of Radon transforms on the other hand.

In the following, we are interested in Crofton type questions for areas in Minkowski and projective Finsler spaces, avoiding smoothness assumptions where possible, and emphasizing the connections to zonoid theory. General references for volumes and areas in Minkowski and Finsler spaces are the book of Thompson ${ }^{50}$ and the article by Álvarez and Thompson ${ }^{12}$. The latter is a highly recommended survey article, giving a thorough introduction to volumes on normed and Finsler spaces. Insisting on the intrinsic approach as it does, it may for many readers be more satisfactory under formal aspects than the following brief ad hoc introduction, which sacrifices the pureness of approach to a more intuitive presentation and to the ease of calculations.

In an $n$-dimensional Finsler space, there is a natural notion of curve length, but no canonical notion of area for $k$-dimensional submanifolds, if $1<k \leq n$. Instead, there are several options. This becomes already clear in the case of a Minkowski space $\left(\mathbb{R}^{n},\|\cdot\|\right)$, which we consider first.

It is natural to assume, and we do this, that any area in $\left(\mathbb{R}^{n},\|\cdot\|\right)$ should 
be invariant under the isometries of the space and thus, in particular, under translations. The $n$-dimensional area, or volume, is thus required to be a translation invariant measure (locally finite, as always) on $\mathbb{R}^{n}$ and hence is uniquely determined up to a constant factor. This factor can be chosen so that the volume of the unit ball $B$ of the norm $\|\cdot\|$ has some desired value. In an analogous way, for $1<k<n$, in every $k$-dimensional subspace a notion of $k$-dimensional volume is uniquely determined up to a factor. This factor, however, depends on the subspace. For that reason, there are many possibilities to define a $k$-dimensional area. The geometric properties of such areas can be very different, as will be seen later.

Since a $k$-dimensional area in a Minkowski space should be determined by its metric and thus by its unit ball, up to the freedom of choosing normalizing factors as described above, the following axioms for such an area, essentially going back to Busemann ${ }^{19}$, are natural. Let $\mathcal{C}^{k}$ denote the set of all $k$-dimensional convex bodies in $\mathbb{R}^{n}$ which are centrally symmetric with respect to the origin. A $k$-normalization is a function

$$
\alpha_{k}: \mathcal{C}^{k} \rightarrow \mathbb{R}^{+}
$$

satisfying the following properties (M1) - (M3):

(M1) $\alpha_{k}$ is invariant under linear transformations of $\mathbb{R}^{n}$,

(M2) $\alpha_{k}$ is continuous (with respect to the Hausdorff metric),

(M3) $\alpha_{k}\left(E_{k}\right)=\kappa_{k}$, if $E_{k}$ is a $k$-dimensional ellipsoid.

If a $k$-normalization $\alpha_{k}$ is given, the induced Minkowskian $k$-area $\alpha_{k}^{B}$ on $\left(\mathbb{R}^{n},\|\cdot\|\right)$ is defined by

$$
\alpha_{k}^{B}(M):=\int_{M} \frac{\alpha_{k}\left(B \cap T_{x} M\right)}{\lambda_{k}\left(B \cap T_{x} M\right)} \lambda_{k}(\mathrm{~d} x)
$$

for any compact $C^{1}$ submanifold $M$ of $\mathbb{R}^{n}$; here $T_{x} M$ is the tangent space of $M$ at $x$ (a subspace of $\mathbb{R}^{n}$, since we have identified $T_{x} \mathbb{R}^{n}$ with $\mathbb{R}^{n}$ ). We have attached the upper index $B$ to $\alpha_{k}^{B}$ since the area depends on both, the $k$-normalization $\alpha_{k}$ and the norm $\|\cdot\|$ with unit ball $B$. Further, we have used the auxiliary Euclidean structure, since this is often convenient for calculations, but the definition is independent of the choice of the Euclidean metric. An intrinsic representation is given by (24) below.

In the special case $k=n-1$, a further axiom plays an important role. To formulate it, we define the scaling function (depending on the auxiliary 
Euclidean metric) by

$$
\sigma_{\alpha, B}(u):=|u| \frac{\alpha_{n-1}\left(B \cap u^{\perp}\right)}{\lambda_{n-1}\left(B \cap u^{\perp}\right)} \quad \text { for } u \in \mathbb{R}^{n} \backslash\{0\} .
$$

The fourth axiom demands:

(M4) $\sigma_{\alpha, B}$ is a norm on $\mathbb{R}^{n}$ (for any $B$ ).

The raison d'être for this axiom is the fact that it is equivalent to the areaminimizing property of flat regions; see Álvarez and Thompson ${ }^{12}$, Section 4 , for the explanation of several versions of this equivalence.

If condition (M4) is satisfied, then $\sigma_{\alpha, B}$ is the support function of a convex body $\mathbf{I}_{B}$. Suppose that also a Minkowskian volume is given, by an $n$-normalization $\alpha_{n}$ as above. Then the body $\mathbb{I}_{B}:=\left(\lambda_{n}(B) / \alpha_{n}(B)\right) \mathbf{I}_{B}$ is called the isoperimetrix of the triple $\left(\alpha_{n-1}, \alpha_{n}, B\right)$. The name comes from the connection with the isoperimetric problem: among all convex bodies of the same volume, precisely the homothets of the isoperimetrix have the smallest Minkowskian surface area (see Thompson ${ }^{50}$ or Álvarez and Thompson ${ }^{12}$ ). The chosen normalization makes $\mathbb{I}_{B}$ independent of the auxiliary Euclidean structure.

Now let $\left(\mathbb{R}^{n}, F\right)$ be a Finsler space. Any Minkowskian notion of $k$-area, defined by a $k$-normalization $\alpha_{k}$, immediately yields a notion of $k$-area of $C^{1}$ submanifolds $M$ in $\mathbb{R}^{n}$, just by extending (20) to

$$
\alpha_{k}^{F}(M):=\int_{M} \frac{\alpha_{k}\left(B_{x} \cap T_{x} M\right)}{\lambda_{k}\left(B_{x} \cap T_{x} M\right)} \lambda_{k}(\mathrm{~d} x) .
$$

There are two special choices of $k$-normalizations $\alpha_{k}$ leading to important notions of areas, which appear particularly natural from different points of view. The first one is the trivial function

$$
\alpha_{k}(K):=\kappa_{k} \quad \text { for all } K \in \mathcal{C}^{k},
$$

leading to the Busemann $k$-area,

$$
\beta_{k}(M):=\int_{M} \frac{\kappa_{k}}{\lambda_{k}\left(B_{x} \cap T_{x} M\right)} \lambda_{k}(\mathrm{~d} x) .
$$

The second one is the function given by

$$
\alpha_{k}(K):=\frac{v p(K)}{\kappa_{k}} \quad \text { for all } K \in \mathcal{C}^{k},
$$

where $v p(K)$ is the volume product of $K$, that is, the product of the Euclidean $k$-dimensional volumes of $K$ and its polar body $K^{o}$, taken in the 
affine hull of $K$; this definition is independent of the choice of the Euclidean metric. This second function yields the Holmes-Thompson $k$-area,

$$
\operatorname{vol}_{k}(M):=\int_{M} \frac{\lambda_{k}\left(B_{x}^{o} \mid T_{x} M\right)}{\kappa_{k}} \lambda_{k}(\mathrm{~d} x) .
$$

Here $\mid E$ denotes orthogonal projection to a subspace $E$, and a result from convex geometry was used to replace $\left(B_{x} \cap E\right)^{o}$ by $B_{x}^{o} \mid E$.

The definitions are also employed for $k=n$, thus

$$
\beta_{n}(M):=\kappa_{n} \frac{\lambda_{n}(M)}{\lambda_{n}(B)}
$$

is the Busemann volume, and

$$
\operatorname{vol}_{n}(M):=\frac{1}{\kappa_{n}} \lambda_{n}\left(B^{o}\right) \lambda_{n}(M)
$$

is the Holmes-Thompson volume of the Borel set $M \subset \mathbb{R}^{n}$.

The exceptional role of these two area notions is explained by the fact that they are disguised areas appearing in other contexts. The Busemann area of a $k$-dimensional submanifold of the Finsler space $\left(\mathbb{R}^{n}, F\right)$ is its $k$-dimensional Hausdorff measure $\mathcal{H}_{F}^{k}$ induced by the metric $d_{F}$. Proofs that the Busemann $k$-area of a rectifiable subset of a smooth or general Finsler space coincides with its $k$-dimensional Hausdorff measure $\mathcal{H}_{F}^{k}$, can be found, in different degrees of generality and with different proofs, in Busemann ${ }^{18}$, Bellettini, Paolini and Venturini ${ }^{15}$, Schneider ${ }^{44}$. The HolmesThompson area of a $k$-dimensional submanifold of a Finsler space is the symplectic (or Liouville) volume of its unit co-disc bundle with respect to the induced Finsler metric, divided by the volume of the Euclidean unit ball of dimension $k$.

With the help of the Hausdorff measure $\mathcal{H}_{F}^{k}$, formula $(21)$ can be replaced by

$$
\alpha_{k}^{F}(M)=\frac{1}{\kappa_{k}} \int_{M} \alpha_{k}\left(B_{x} \cap T_{x} M\right) \mathcal{H}_{F}^{k}(\mathrm{~d} x),
$$

as shown in Schneider ${ }^{44}$. This representation is intrinsic, that is, it no longer involves the auxiliary Euclidean metric.

In a Minkowski space (of dimension $n$, with unit ball $B$ ) both, the Busemann $(n-1)$-area and the Holmes-Thompson $(n-1)$-area, satisfy axiom (M4). Denoting the scaling functions of the Busemann area and the Holmes-Thompson area by $\sigma_{B}^{\mathrm{BU}}$ and $\sigma_{B}^{H T}$, respectively, and the isoperimet- 
rices (with respect to the corresponding volumes) by $\mathbb{I}_{B}^{\mathrm{BU}}$ and $\mathbb{I}_{B}^{\mathrm{HT}}$, respectively, we have

$$
\sigma_{B}^{\mathrm{BU}}(u)=\frac{\kappa_{n-1}}{\lambda_{n-1}\left(B \cap u^{\perp}\right)}, \quad u \in S^{n-1}
$$

and

$$
\sigma_{B}^{\mathrm{HT}}(u)=\frac{\lambda_{n-1}\left(B^{\circ} \mid u^{\perp}\right)}{\kappa_{n-1}}, \quad u \in S^{n-1} .
$$

Thus, the isoperimetrices are given by

$$
\mathbb{I}_{B}^{\mathrm{BU}}=\frac{\kappa_{n-1}}{\kappa_{n}} \lambda_{n}(B) \mathrm{I}^{o} B
$$

where $\mathrm{I}$ is the intersection body operator and $\mathrm{I}^{o} B:=(\mathrm{I} B)^{\circ}$ is the polar intersection body of $B$, and

$$
\mathbb{I}_{B}^{\mathrm{HT}}=\frac{\kappa_{n}}{\kappa_{n-1}} \frac{1}{\lambda\left(B^{\circ}\right)} \Pi B^{o},
$$

where $\Pi$ is the projection body operator. For information on intersection and projection bodies, we refer to Schneider $^{40}$ and particularly to Gardner $^{28}$.

\section{Nonexistence and Existence of (Positive) Crofton Measures}

Crofton measures will now first be studied in a Minkowski space $\left(\mathbb{R}^{n},\|\cdot\|\right)$, with unit ball $B$. We assume that $k \in\{2, \ldots, n-1\}$ and that a Minkowskian area defined by a $k$-normalization $\alpha_{k}$ satisfying (M1) - (M3), and (M4) in the case $k=n-1$, is given. By a Crofton measure for the area $\alpha_{k}^{B}$ we understand a translation invariant measure $\varphi_{n-k}$ on the affine Grassmannian $A(n, n-k)$ that satisfies

$$
\int_{A(n, n-k)} \operatorname{card}(E \cap K) \varphi_{n-k}(\mathrm{~d} E)=\alpha_{k}^{B}(K)
$$

for every $k$-dimensional convex body $K$. Thus, in a Minkowski space, we make the translation invariance part of the definition of a Crofton measure. Further, we require the validity of the Crofton type formula (27) only for $k$-dimensional convex bodies $K$. The existence or nonexistence of Crofton measures is already decided in this simple case, and if a Crofton measure exists, then it will later be possible to prove Crofton type formulas for much more general sets. 
The question for the existence of Crofton measures for general Minkowskian (and even more general affine) areas goes back to Busemann ${ }^{19}$, and he has obtained, in a weaker form, the following criterion. Let $G(n, j)$ denote the Grassmannian of $j$-dimensional linear subspaces of $\mathbb{R}^{d}$. For $E \in G(n, k)$ and $L \in G(n, n-k)$ we denote by $[E, L]$ the absolute $k$-dimensional determinant of the orthogonal projection from $E$ to $L^{\perp}$. A proof of the following lemma can be found in Schneider and Wieacker ${ }^{48}$.

Lemma 5.1. A Crofton measure for $\alpha_{k}^{B}$ exists if and only if there is a finite measure $\psi$ on $G(n, n-k)$ such that

$$
\frac{\alpha_{k}(B \cap E)}{\lambda_{k}(B \cap E)}=\int_{G(n, n-k)}[E, L] \psi(\mathrm{d} L) \quad \text { for } E \in G(n, k) \text {. }
$$

In the case $k=n-1$, the left-hand side of (28) is the value of the scaling function at the unit normal vector of the $(n-1)$-dimensional linear subspace $E$. In this case, we can replace the integration over $G(n, 1)$ by an integration over the unit sphere $S^{n-1}$. Moreover, since we have assumed (M4), the scaling function is the support function of the convex body $\mathbf{I}_{B}$, a multiple of the isoperimetrix. Equation (28) is thus equivalent to

$$
h\left(\mathbf{I}_{B}, u\right)=\int_{S^{n-1}}|\langle u, v\rangle| \varphi(\mathrm{d} v) \quad \text { for } u \in \mathbb{R}^{n},
$$

with a finite even measure $\varphi$ on $S^{n-1}$. Hence, we obtain the following result. The isoperimetrix and the body $\mathrm{I}^{o} B$ occurring here depend on additional data (a volume, or the Euclidean metric), but only up to a factor, which is irrelevant.

Theorem 5.1. For a Minkowskian $(n-1)$-area a Crofton measure (a positive Crofton measure) exists if and only if the isoperimetrix is a generalized zonoid (a zonoid).

For the Busemann $(n-1)$-area a Crofton measure (a positive Crofton measure) exists if and only if $\mathrm{I}^{\circ} B$, the polar intersection body of the unit ball, is a generalized zonoid (a zonoid).

For the Holmes-Thompson $(n-1)$-area, a positive Crofton measure always exists.

The latter assertion follows from (26) and the fact that projection bodies are zonoids, hence the zonoid equation (29) for the support function of $\mathbf{I}_{B}$ can be solved with a positive measure $\varphi$. 
By Theorem 5.1, the existence of Crofton measures for Minkowskian $(n-1)$-areas is closely connected with the theory of generalized zonoids. We will now exploit this connection.

Theorem 5.2. There exist Minkowski spaces, for example $\ell_{\infty}^{n}$ and $\ell_{1}^{n}$, for which the only Minkowskian $(n-1)$-area admitting a positive Crofton measure is the Holmes-Thompson area (up to a factor).

This was proved in Schneider ${ }^{41}$. By Theorem 5.1, the proof reduces to a question on zonoids, of the following type. The unit ball of the Minkowski space in question determines two centred polytopes $P$ and $Q$, where $Q \subset P$, $\mu Q \not \subset P$ for $\mu>1$, and $P$ is a zonotope. One has to show that a zonoid $Z$ satisfying $Q \subset Z \subset P$ necessarily coincides with $P$. For example, if $n=3$, then the pair $(Q, P)$ in the case of $\ell_{\infty}^{3}$ is the pair (octahedron, rhombic dodecahedron), and in the case of $\ell_{1}^{3}$ the pair (cuboctahedron, cube). That assertions about general Minkowskian surface areas are possible at all, is due, roughly speaking, to Axiom (M1) and the fact that for the cube and the cross-polytope many central hyperplane sections are linearly equivalent.

In the following, we use the Banach-Mazur distance to topologize the set of all $n$-dimensional Minkowski spaces (more precisely, the set of isometry classes of $n$-dimensional Minkowski spaces).

Theorem 5.3. In every Minkowski space of sufficiently large dimension $n$ which is sufficiently close to $\ell_{\infty}^{n}$, there exists no positive Crofton measure for the Busemann $(n-1)$-area.

The basic ideas of the proof, given in Schneider ${ }^{42}$, are the following. According to Theorem 5.1, we have to show that for the spaces in question the polar intersection body $\mathrm{I}^{o} B$ of the unit ball $B$ is not a zonoid. For this, we need a lemma expressing that cross-polytopes are very far from zonoids. Let $\left(e_{1}, \ldots, e_{n}\right)$ be an orthonormal basis of $\mathbb{R}^{n} ;$ then $Q:=\operatorname{conv}\left\{ \pm e_{1}, \ldots, \pm e_{n}\right\}$ is a cross-polytope.

Lemma 5.2. If

$$
0<\gamma<\gamma_{n}:=2^{-(n-1)} n\left(\begin{array}{c}
n-1 \\
\left\lfloor\frac{n-1}{2}\right\rfloor
\end{array}\right)
$$

and $K$ is a convex body with

$$
Q \subset K \subset \gamma Q
$$

then $K$ is not a zonoid. 
As a side remark, we point out that $\gamma_{n} \sim \sqrt{2 n / \pi}$ for $n \rightarrow \infty$.

The space $\ell_{\infty}^{n}$ can be identified with $\mathbb{R}^{n}$ with unit ball given by the cube $C$ with vertices $\pm e_{1} \pm \cdots \pm e_{n}$. We consider the multiple $\alpha \mathrm{I}^{\circ} C$ of the polar intersection body of $C$ that satisfies $h\left(\alpha \mathrm{I}^{\circ} C, e_{1}\right)=1$. Since $\alpha \mathrm{I}^{\circ} C$ has the same symmetries as $C$, it follows that

$$
Q \subset \alpha \mathrm{I}^{\circ} C .
$$

Suppose that also

$$
\alpha \mathrm{I}^{\circ} C \subset \operatorname{int} \lambda_{n} Q
$$

If this holds, then $Q \subset \operatorname{int} a \mathrm{I}^{\circ} C \subset \operatorname{int} \lambda Q$ for suitable numbers $a>1$ and $\lambda<\lambda_{n}$. Since the intersection body operator is continuous, there is a neighbourhood $U$ of the cube such that for all Minkowskian unit balls $B \subset U$ the polar intersection body also satisfies

$$
Q \subset \operatorname{int} a \mathrm{I}^{o} B \subset \operatorname{int} \lambda Q .
$$

By Lemma $5.2, \mathrm{I}^{\circ} B$ is not a zonoid, hence there is no positive Crofton measure for the Busemann area in the Minkowski space with unit ball $B$.

To establish (30), we set $z=e_{1}+\cdots+e_{n}$ and show that

$$
h\left(\alpha \mathrm{I}^{\circ} C, z\right)<\lambda_{n} h(Q, z) .
$$

If this is proved, then (30) follows by symmetry. Now

$$
h\left(\alpha \mathrm{I}^{o} C, z\right)=\frac{\sqrt{n}}{S(n)},
$$

where $S(n)$ denotes the $(n-1)$-volume of the intersection of the cube $\frac{1}{2} C$ with a hyperplane through its centre and orthogonal to a main diagonal. It is known (see, for example, Chakerian and Logothetti ${ }^{23}$ ) that

$$
S(n)=\frac{2}{n} \sqrt{\pi} \int_{0}^{\infty}\left(\frac{\sin x}{x}\right)^{n} \mathrm{~d} x \rightarrow \sqrt{6 / \pi}
$$

for $n \rightarrow \infty$. From this, it follows that (31) is satisfied for all sufficiently large dimensions $n$.

Theorem 5.4. There exist Minkowski spaces arbitrarily close to the Euclidean space $\ell_{2}^{n}$ in which there exists no positive Crofton measure for the Busemann $(n-1)$-area. 
To obtain this result, one has to construct convex bodies, arbitrarily close to the Euclidean unit ball $B^{n}$, for which the polar intersection body is not a zonoid. In Schneider ${ }^{42}$, this is achieved as follows. Let $u, z \in S^{n-1}$ be orthogonal unit vectors, and let $\epsilon>0$. Define

$$
B_{0}:=\operatorname{conv}\left(B^{n} \cup(1+\epsilon)\left(B^{n} \cap u^{\perp}\right)\right)
$$

and $B:=B_{0}+\epsilon \operatorname{conv}\{-z, z\}$. By computing the directional derivatives of the section volume function $v \mapsto \lambda_{n-1}\left(B \cap v^{\perp}\right)$ at $u$, one can deduce that the face $F\left(\mathrm{I}^{\circ} B, u\right)$ of the polar intersection body of $B$ with outer normal vector $u$ contains an $(n-1)$-dimensional ball as a summand.

Further, the body $B$ has a cylindrical part. This implies that there is a neighbourhood $U$ of the vector $z$ such that

$$
h\left(\mathrm{I}^{o} B, y\right)=h\left(\mathrm{I}^{o} B, z\right)\langle y, z\rangle \quad \text { for } y \in U .
$$

This means that the body $\mathrm{I}^{o} B$ has a vertex $z_{0}$ with outer normal vector $z$.

Now assume that $\mathrm{I}^{\circ} B$ were a zonoid. Then the face $F\left(\mathrm{I}^{\circ} B, u\right)$ is a summand of $\mathrm{I}^{\circ} B$. In particular, $\mathrm{I}^{\circ} B$ has a summand $K$ which is an $(n-1)$ dimensional ball. There is a translate $K^{\prime}$ of $K$ such that $z_{0} \in K^{\prime} \subset \mathrm{I}^{\circ} B$. But this is not possible, since $z_{0}$ is a vertex of $\mathrm{I}^{o} B$. Thus $\mathrm{I}^{o} B$ cannot be a zonoid.

In general, it is difficult to verify that a given convex body is not a zonoid, except in the trivial case where it has a face that is not centrally symmetric. This difficulty is one obstacle for a proof of the following:

Conjecture. In the space of $n$-dimensional Minkowski spaces, there is a dense subset of spaces in which there is no positive Crofton measure for the Busemann $(n-1)$-area.

On the other hand, it would be rash to conjecture that a positive Crofton measure for the Busemann area existed only in Euclidean spaces.

Theorem 5.5. There exist Minkowski spaces arbitrarily close to $\ell_{2}^{n}$, but not Euclidean, in which there does exist a positive Crofton measure for the Busemann $(n-1)$-area.

This is proved in Schneider ${ }^{42}$, by smooth perturbation of the Euclidean unit ball $B^{n}$. It is shown that this can be done in such a way that the obtained body $B_{\epsilon}$ is convex, centrally symmetric and smooth, but not an ellipsoid, and that for the support function of the polar intersection body $\mathrm{I}^{o} B_{\epsilon}$, the zonoid equation still has a positive solution. This means that $\mathrm{I}^{\circ} B_{\epsilon}$ 
is a zonoid, hence in the Minkowski space with unit ball $B_{\epsilon}$, the Busemann $(n-1)$-area admits a positive Crofton measure.

Theorem 5.5 gives a positive answer to the third of the open problems in Chakerian ${ }^{22}$.

One consequence of the preceding results is the conclusion that the Busemann $(n-1)$-area, although very natural, being a Hausdorff measure, is not suitable for integral geometry, since for it not even the simplest Crofton formulas with positive measures exist in all Minkowski spaces. Even more restrictions arise in Finsler spaces. In a Minkowski space, under strong smoothness assumptions, the zonoid equation (29) for the support function of the isoperimetrix $\mathbb{I}_{B}^{\mathrm{BU}}$ has a solution, hence there exists a signed Crofton measure for the Busemann $(n-1)$-area. In a projective Finsler space, even smoothness assumptions are not sufficient to obtain Crofton formulas for the Busemann area. An example to this effect was constructed by Álvarez and Berck ${ }^{6}$.

For the Holmes-Thompson area, the situation is much better. This is already seen from the last part of Theorem 5.1, asserting that in every $n$-dimensional Minkowski space a positive Crofton measure exists for the Holmes-Thompson $(n-1)$-area $\operatorname{vol}_{n-1}$. For the lower-dimensional HolmesThompson areas $\operatorname{vol}_{k}$, the following holds.

Theorem 5.6. If in an n-dimensional Minkowski space there exists a Crofton measure (a positive Crofton measure) for the norm, then there also exists a Crofton measure (a positive Crofton measure) for $\operatorname{vol}_{k}, k=$ $2, \ldots, n-2$.

This follows from the first part of Theorem 2.1 and the construction in Section 7. There are two main cases where the assumption of Theorem 5.6 is satisfied:

- If the norm $\|\cdot\|=h\left(B^{o}, \cdot\right)$ is sufficiently smooth, then $B^{\circ}$ is a generalized zonoid, hence a Crofton measure for vol $_{1}$ exists.

- If the Minkowski space $(\mathbb{R},\|\cdot\|)$ is hypermetric then, by Theorem 2.2 , a positive Crofton measure for vol $_{1}$ exists.

Under either of these two assumptions, smooth or hypermetric, the existence of Crofton measures for the Holmes-Thompson areas of all dimensions extends to projective Finsler spaces, and Crofton formulas for quite general subsets can be proved. For this, more information on generalized zonoids is helpful, and this will be collected in the next section. 


\section{More on Generalized Zonoids}

Let $Z \subset \mathbb{R}^{n}$ be a generalized zonoid with centre 0 . Thus, the support function of $Z$ has an integral representation

$$
h(Z, \xi)=\int_{S^{n-1}}|\langle\xi, u\rangle| \rho(\mathrm{d} u)
$$

with a finite (signed) measure $\rho$ on the sphere $S^{n-1}$. This equation can be interpreted as giving half the one-dimensional volume of the orthogonal projection of $Z$ on to the linear subspace spanned by $\xi$. There is an extension to volumes of higher-dimensional projections. By $L\left(u_{1}, \ldots, u_{k}\right)$ and $\left[u_{1}, \ldots, u_{k}\right]$ we denote, respectively, the linear subspace spanned by the vectors $u_{1}, \ldots, u_{k}$ and the $k$-dimensional (Euclidean) volume of the parallelepiped spanned by these vectors. Let $k \in\{1, \ldots, n\}$ and $E \in A(n, k)$. Then

$$
\begin{aligned}
& \lambda_{k}(Z \mid E) \\
& =\frac{2^{k}}{k !} \int_{S^{n-1}} \cdots \int_{S^{n-1}}\left[E, L\left(u_{1}, \ldots, u_{k}\right)^{\perp}\right]\left[u_{1}, \ldots, u_{k}\right] \rho\left(\mathrm{d} u_{1}\right) \cdots \rho\left(\mathrm{d} u_{k}\right) .
\end{aligned}
$$

The proof given by Weil ${ }^{52}$ holds also for signed measures. Equation (33) can be written in a more concise form, after defining the "projection generating measure' $\rho^{(k)}$ on $G(n, k)$ by

$$
\begin{aligned}
& \rho^{(k)}(A) \\
& :=c_{k} \int_{S^{n-1}} \cdots \int_{S^{n-1}} \mathbf{1}_{A}\left(L\left(u_{1}, \ldots, u_{k}\right)\right)\left[u_{1}, \ldots, u_{k}\right] \rho\left(\mathrm{d} u_{1}\right) \cdots \rho\left(\mathrm{d} u_{k}\right)
\end{aligned}
$$

for Borel sets $A \subset G(n, k)$, with $c_{k}$ given by

$$
c_{k}:=\frac{2^{k}}{k ! \kappa_{k}} \text {. }
$$

Then (33) takes the form

$$
\lambda_{k}(Z \mid E)=\kappa_{k} \int_{G(n, k)}\left[E, L^{\perp}\right] \rho^{(k)}(\mathrm{d} L) \quad \text { for } E \in G(n, k) .
$$

The definition (34) essentially goes back to Matheron ${ }^{35}$, p. 101; later uses of the projection generating measure begin with Goodey and Weil ${ }^{30}$.

If we define $\rho_{(n-k)}$ as the image measure of $\rho^{(k)}$ under the map $L \mapsto L^{\perp}$ from $G(n, k)$ to $G(n, n-k)$, then (35) can be written in the form

$$
\frac{\lambda_{k}(Z \mid E)}{\kappa_{k}}=\int_{G(n, n-k)}[E, L] \rho_{(n-k)}(\mathrm{d} L) \quad \text { for } E \in G(n, k) \text {. }
$$


The case $k=n-1$ has a special feature, since the measure $\rho_{(n-1)}$ is related to the area measure $S_{n-1}(Z, \cdot)$ of $Z$. For $u \in S^{n-1}$ we have, by (36) and a well-known representation of the projection volume,

$$
\begin{aligned}
\frac{\lambda_{k}\left(Z \mid u^{\perp}\right)}{\kappa_{n-1}} & =\int_{G(n, 1)}\left[u^{\perp}, L\right] \rho_{(1)}(\mathrm{d} L) \\
& =\frac{1}{2 \kappa_{n-1}} \int_{S^{n-1}}|\langle u, v\rangle| S_{n-1}(Z, \mathrm{~d} v) .
\end{aligned}
$$

From the uniqueness result for the zonoid equation, it follows that the measure $2 \kappa_{n-1} \rho_{(1)}$ is the image measure of $S_{n-1}(Z, \cdot)$ under the map $u \mapsto$ $L(u)$ from $S^{n-1}$ to $G(n, 1)$.

Now we assume that $Z$ has a representation

$$
h(Z, \xi)=\int_{S^{n-1}}|\langle\xi, u\rangle| g(u) \sigma(\mathrm{d} u)
$$

with a continuous function $g$. Let $s_{n-1}(Z, u)$ be the product of the principal radii of curvature of $Z$ at the boundary point with outer unit normal vector $u$. The following formula, proved by Weil ${ }^{51}$, Satz 7 , will be needed:

$$
\begin{aligned}
s_{n-1}(Z, u)= & \frac{2^{n-1}}{(n-1) !} \int_{s_{u}} \ldots \int_{s_{u}}\left[u_{1}, \ldots, u_{n-1}\right]^{2} g\left(u_{1}\right) \cdots g\left(u_{n-1}\right) \\
& \times \sigma_{u}\left(\mathrm{~d} u_{1}\right) \cdots \sigma_{u}\left(\mathrm{~d} u_{n-1}\right) .
\end{aligned}
$$

Here $\sigma_{u}$ is the $(n-2)$-dimensional spherical Lebesgue measure on the sphere $s_{u}:=S^{n-1} \cap u^{\perp}$.

\section{Crofton Formulas in Smooth Projective Finsler Spaces}

The results on generalized zonoids have immediate applications to the existence of Crofton measures for Holmes-Thompson areas. Let us first consider a Minkowski space $\left(\mathbb{R}^{n},\|\cdot\|\right)$ for which the polar unit ball $B^{o}$ is a generalized zonoid. Then there is a representation

$$
h\left(B^{o}, \xi\right)=\int_{S^{n-1}}|\langle\xi, u\rangle| \rho(\mathrm{d} u)
$$

with a finite measure $\rho$. For $k \in\{1, \ldots, n-1\}$, let $\rho_{(n-k)}$ be the corresponding measure on $G(n, n-k)$ as defined in Section 6. By Lemma 5.1, (36) is precisely the condition which ensures that a Crofton measure exists for the Holmes-Thompson $k$-area $\operatorname{vol}_{k}$. This Crofton measure $\eta_{n-k}$ is defined by

$$
\int_{A(n, n-k)} f \mathrm{~d} \eta_{n-k}:=\int_{G(n, n-k)} \int_{L^{\perp}} f(L+t) \lambda_{k}(\mathrm{~d} t) \rho_{(n-k)}(\mathrm{d} L)
$$


for nonnegative measurable functions $f$ on $A(n, n-k)$. In terms of parameterized hyperplanes, it can be represented by

$$
\begin{aligned}
\int_{A(n, n-k)} f \mathrm{~d} \eta_{n-k}= & c_{k} \int_{S^{n-1}} \ldots \int_{S^{n-1}} \int_{\mathbb{R}} \ldots \int_{\mathbb{R}} f\left(H_{u_{1}, t_{1}} \cap \cdots \cap H_{u_{k}, t_{k}}\right) \\
& \times \mathrm{d} t_{1} \cdots \mathrm{d} t_{k} \rho\left(\mathrm{d} u_{1}\right) \cdots \rho\left(\mathrm{d} u_{k}\right) .
\end{aligned}
$$

Thus, $\eta_{n-k}$ is the image measure of $c_{k}\left(\eta_{n-1}\right)^{\otimes k}$ under the intersection map

$$
\left(H_{1}, \ldots, H_{k}\right) \mapsto H_{1} \cap \cdots \cap H_{k}
$$

from the set of independent $k$-tuples of hyperplanes to $A(n, n-k)$.

This existence and representation of Crofton measures for HolmesThompson areas was first obtained in Schneider and Wieacker ${ }^{48}$, for hypermetric Minkowski spaces. In that case, the Crofton measures are positive. The proofs given in Schneider and Wieacker ${ }^{48}$ carry over, without change, to Minkowski spaces whose polar unit balls are generalized zonoids, thus, in particular, to spaces with sufficiently smooth norms. The Crofton measures are then signed measures. However, the line measure $\eta_{1}$ is always a positive measure. This follows from the remark in Section 6 concerning $\rho_{(1)}$. It shows that the line measure $\eta_{1}$ given by (39) can also be represented by

$$
\int_{A(n, 1)} f \mathrm{~d} \eta_{1}=\frac{1}{2 \kappa_{n-1}} \int_{S^{n-1}} \int_{u^{\perp}} f(L(u)+x) \lambda_{n-1}(\mathrm{~d} x) S_{n-1}\left(B^{o}, \mathrm{~d} u\right)
$$

and thus is positive. This measure on the space of lines can be defined in general Minkowski spaces; it appeared already in Busemann ${ }^{19}$ and later in El-Ekhtiar ${ }^{25}$.

The general Crofton formulas obtained in Schneider and Wieacker ${ }^{48}$, and the methods to prove them, extend to smooth projective Finsler spaces, and will now be formulated in this generality.

Let $\left(\mathbb{R}^{n}, F\right)$ be a smooth projective Finsler space. We combine Pogorelov's approach with the preceding one. In particular, the representation (19) of the local polar unit ball $B_{x}^{o}$ at $x \in \mathbb{R}^{n}$ replaces now the representation (38). The (signed) measure $\eta_{n-1}=\eta$ is defined by (18). Following the procedure above, for $k \in\{2, \ldots, n-1\}$ we define $\eta_{n-k}$ as the image measure of $c_{k}\left(\eta_{n-1}\right)^{\otimes k}$ under the intersection map. Explicitly, this means that

$$
\begin{aligned}
& \int_{A(n, n-k)} f \mathrm{~d} \eta_{n-k} \\
& =c_{k} \int_{S^{n-1}} \cdots \int_{S^{n-1}} \int_{\mathbb{R}} \cdots \int_{\mathbb{R}} f\left(H_{u_{1}, t_{1}} \cap \cdots \cap H_{u_{k}, t_{k}}\right) \\
& \quad \times g\left(u_{1}, t_{1}\right) \cdots g\left(u_{k}, t_{k}\right) \mathrm{d} t_{1} \cdots \mathrm{d} t_{k} \sigma\left(\mathrm{d} u_{1}\right) \cdots \sigma\left(\mathrm{d} u_{k}\right) .
\end{aligned}
$$


With these Crofton measures, we can formulate general Crofton formulas for Holmes-Thompson areas. They are general in two respects: the submanifolds need not be smooth, and submanifold and intersecting flats need not be of complementary dimensions.

In the following, we denote by $\mathcal{H}^{k}$ the $k$-dimensional Hausdorff measure that is induced by the auxiliary Euclidean metric on $\mathbb{R}^{n}$, for $k \in\{0, \ldots, n\}$. The restriction of this outer measure to the Borel sets is $\sigma$-additive. A set $M \subset \mathbb{R}^{n}$ is called $\left(\mathcal{H}^{k}, k\right)$-rectifiable if $\mathcal{H}^{k}(M)<\infty$ and there exist Lipschitz maps $f_{i}: \mathbb{R}^{k} \rightarrow \mathbb{R}^{n}, i \in \mathbb{N}$, such that $\mathcal{H}^{k}\left(K \backslash \bigcup_{i \in \mathbb{N}} f_{i}\left(\mathbb{R}^{k}\right)\right)=0$. This notion does not depend on the choice of the Euclidean metric. For a $\left(\mathcal{H}^{k}, k\right)$-rectifiable Borel set, we can define the $k$-dimensional HolmesThompson area by extending (23),

$$
\operatorname{vol}_{k}(M):=\frac{1}{\kappa_{k}} \int_{M} \lambda_{k}\left(B_{x}^{o} \mid T_{x} M\right) \mathcal{H}^{k}(\mathrm{~d} x) .
$$

The approximate tangent space $T_{x} M$ exists and is unique for $\mathcal{H}^{k}$-almost all $x \in M$; it is a measurable function of $x$.

With these definitions, a general Crofton formula of type (1) can be obtained. Moreover, it can be extended to the case where the submanifold and the intersecting flats are no longer of complementary dimensions.

Theorem 7.1. Let $\mathbb{R}^{n}$ be endowed either with a norm for which the polar unit ball is a generalized zonoid, or with a smooth projective Finsler metric. Let $M \subset \mathbb{R}^{n}$ be a $\left(\mathcal{H}^{k}, k\right)$-rectifiable Borel set, $k \in\{1, \ldots, n\}$. Then

$$
\int_{A(n, n-k)} \operatorname{card}(E \cap M) \eta_{n-k}(\mathrm{~d} E)=\operatorname{vol}_{k}(M) .
$$

More generally, if $j \in\{n-k, \ldots, n-1\}$, then

$$
\int_{A(n, j)} \operatorname{vol}_{k+j-n}(E \cap M) \eta_{j}(\mathrm{~d} E)=\frac{c_{k+j-n} c_{n-j}}{c_{k}} \operatorname{vol}_{k}(M) .
$$

Theorem 7.1 can be viewed as giving a positive answer, for projective Finsler spaces, to the first of the three open problems formulated by Chakerian ${ }^{22}$.

As mentioned, formula (44) for hypermetric Minkowski spaces was first proved in Schneider and Wieacker ${ }^{48}$. Formula (43) and the case $k=n-1$ of (44) for smooth submanifolds of smooth projective Finsler spaces are due to Álvarez and Fernandes ${ }^{7}$. Their proof employs the symplectic structure on the space of geodesics of a projective Finsler space. The theme of Crofton formulas in smooth projective Finsler spaces was taken up in Álvarez and 
Fernandes ${ }^{8}$ and in the thesis of Fernandes ${ }^{26}$, where now part of the methods is closer to those in Schneider and Wieacker ${ }^{48}$, in so far as connections to Fourier transforms of norms, and thus to cosine transforms and the zonoid equation, are used. In Fernandes ${ }^{26}$ and in Álvarez and Fernandes ${ }^{10}$, the topic is considerably expanded, and the role of double fibrations and the Gelfand transform is emphasized. This approach to Crofton formulas is neatly set out in the survey article by Álvarez and Fernandes ${ }^{9}$.

The general formula (44) for smooth projective Finsler spaces was proved in Schneider ${ }^{44}$.

About the line measure $\eta_{1}$ we remark that the representation (40) has now the counterpart

$$
\int_{A(n, 1)} f \mathrm{~d} \eta_{1}=\frac{1}{2 \kappa_{n-1}} \int_{S^{n-1}} \int_{u^{\perp}} f(L(u)+x) s_{n-1}\left(B_{x}^{o}, u\right) \lambda_{n-1}(\mathrm{~d} x) \sigma(\mathrm{d} u) .
$$

The proof uses (37) and is found in Schneider ${ }^{43}$, p. 95. In other words, a density $\delta$ of the line measure $\eta_{1}$ with respect to the motion invariant measure on $A(n, 1)$ is given by $\delta(L(u)+x)=s_{n-1}\left(B_{x}^{o}, u\right)$. Since this density is nonnegative, $\eta_{1}$ is again a positive measure.

The form of the density $\delta$ has the following consequence. If $x$ and $y$ lie on a line with direction $u$, then $L(u)+x=L(u)+y$ and hence $s_{n-1}\left(B_{x}^{o}, u\right)=s_{n-1}\left(B_{y}^{o}, u\right)$. This should not come as a surprise; it is implied by the remark following Theorem 3.1.

\section{Crofton Measures in General Projective Finsler Spaces}

Crofton measures for Holmes-Thompson areas in general Finsler spaces, without smoothness assumptions, have so far only been found in cases where the measures turn out to be positive.

Let $\left(\mathbb{R}^{n}, F\right)$ be a hypermetric projective Finsler space. By Theorem 3.2, due to Alexander, there exists a positive measure $\eta$ on the space $A(n, n-1)$ of hyperplanes such that

$$
\operatorname{vol}_{1}(S)=\int_{A(n, n-1)} \operatorname{card}(E \cap S) \eta(\mathrm{d} E)
$$

for every line segment $S$; here $\operatorname{vol}_{1}$ is the curve length in the Finsler space. This result can be extended to the higher dimensional Holmes-Thompson areas, with positive Crofton measures. Let $k \in\{1, \ldots, n-1\}$. Motivated by the procedure of Schneider and Wieacker ${ }^{48}$ in hypermetric Minkowski spaces, we define a positive measure $\eta_{n-k}$ on the space $A(n, n-k)$ of $(n-k)$ - 
flats as the image measure, under the intersection map

$$
\left(H_{1}, \ldots, H_{k}\right) \mapsto H_{1} \cap \cdots \cap H_{k},
$$

of the product measure $c_{k} \eta^{\otimes k}$, restricted to the set of $k$-tuples $\left(H_{1}, \ldots, H_{k}\right)$ with $(n-k)$-dimensional intersection.

Theorem 8.1. For $k \in\{1, \ldots, n-1\}$, the positive measure $\eta_{n-k}$, defined in the hypermetric projective Finsler space $\left(\mathbb{R}^{n}, F\right)$, satisfies

$$
\operatorname{vol}_{k}(M)=\int_{A(n, n-1)} \operatorname{card}(E \cap M) \eta_{n-k}(\mathrm{~d} E)
$$

for every $k$-dimensional compact convex set $M$.

This was proved in Schneider ${ }^{43}$. Theorem 8.1 gives an answer to the second open problem of Chakerian ${ }^{22}$. The extension to more general sets $M$ and to formulas of type (44) has not been investigated. The validity of (47) for $k$-dimensional compact convex sets already suffices to obtain the uniqueness of the measure $\eta_{n-k}$ for $k=1$ (shown in Alexander ${ }^{2}$ ) and $k=n-1$ (shown in Schneider ${ }^{43}$ ). For $k \in\{2, \ldots, n-2\}$, uniqueness fails.

The proof of Theorem 8.1 uses approximation. By results of Pogorelov ${ }^{36}$ and Szabó ${ }^{49}$, there is for every $\epsilon>0$ a Finsler metric $F_{\epsilon}$ on $\mathbb{R}^{n}$ such that $\left(\mathbb{R}^{n}, F_{\epsilon}\right)$ is a smooth projective Finsler space and $\lim _{\epsilon \rightarrow 0} F_{\epsilon}=F$ uniformly on every compact set. The Finsler metric $F_{\epsilon}$ is hypermetric, too (as noted by Alexander), hence every norm $F_{\epsilon}(x, \cdot)$ is hypermetric. Therefore, every local polar unit ball $B_{x}^{o}$ is a zonoid, which implies that the function $g$ appearing in (18) is nonnegative. Hence, the measure $\eta_{\epsilon}$ defined by (18) for the smooth Finsler metric $F_{\epsilon}$ is positive. The same holds for the derived measures $\left(\eta_{\epsilon}\right)_{n-k}$. One can then let $\epsilon$ tend to 0 and work with vaguely converging subsequences of the corresponding sequences of positive measures to complete the proof of Theorem 8.1.

The good properties of the hypermetric projective Finsler spaces raise the following open question:

Problem. Are the only hypermetric Hilbert geometries of dimension $n>$ 2 the hyperbolic geometries? Equivalently: Let $K$ be an $n$-dimensional convex body $(n>2)$ with the property that $(K-x)^{o}-(K-x)^{o}$ is a zonoid for every $x \in \operatorname{int} K$. Must $K$ be an ellipsoid?

The measure $\left(\eta_{\epsilon}\right)_{1}$ constructed above is always positive, by (45), even if the spaces are not hypermetric. Therefore, the approximation procedure can be modified to yield the following result (details are in Schneider ${ }^{43}$ ). 
Theorem 8.2. In a general projective Finsler space $\left(\mathbb{R}^{n}, F\right)$, there always exists a unique positive measure $\eta_{1}$ on the space $A(n, 1)$ of lines such that

$$
\operatorname{vol}_{k}(M)=\int_{A(n, n-1)} \operatorname{card}(E \cap M) \eta_{1}(\mathrm{~d} E)
$$

for every $(n-1)$-dimensional compact convex set $M$.

The existence and uniqueness of the positive line measure $\eta_{1}$ can be extended, without essential changes of the method, to general projective Finsler spaces $(C, F)$, where $C \subset \mathbb{R}^{n}$ is a bounded open convex subset. For Minkowski spaces, the line measure is explicitly given by (40). This case includes also non-smooth projective Finsler spaces. For example, one can read off from (40) on which sets of lines the line measure is concentrated if the unit ball is a polytope. Besides Minkowski spaces, the only nonsmooth cases where the line measure $\eta_{1}$ is explicitly known are the Hilbert geometries in a convex polygon in the plane; see Alexander ${ }^{2}$ and, in more detail, Alexander, Berg and Foote ${ }^{3}$. The higher-dimensional case requires a different approach. In Schneider ${ }^{45}$, the case of the Hilbert geometry in an $n$-dimensional polytope is investigated. It turns out that the line measure is concentrated on the set of lines meeting two disjoint faces of the polytope whose dimensions add up to $n-1$. This set of lines has dimension $n-1$, whereas $A(n, 1)$ itself has dimension $2(n-1)$.

\section{References}

1. R. Alexander, Planes for which the lines are the shortest paths between points. Illinois J. Math. 22 (1978), 177-190.

2. R. Alexander, Zonoid theory and Hilbert's fourth problem. Geom. Dedicata 28 (1988), 199-211.

3. R. Alexander, I.D. Berg, R. Foote, Integral-geometric formulas for perimeter in $\mathbb{S}^{2}, \mathbb{H}^{2}$, and Hilbert planes. Rocky Mountain J. Math. (to appear).

4. J.C. Álvarez Paiva, Hilbert's fourth problem in two dimensions I. In: "Mass Selecta: Teaching and Learning Advanced Undergraduate Mathematics" (S. Katok, A. Sossinsky, S. Tabachnikov, eds.), pp. 165-183, Amer. Math. Soc., Providence, RI 2003.

5. J.C. Álvarez Paiva, Symplectic geometry and Hilbert's fourth problem. J. Differential Geom. (to appear).

6. J.C. Álvarez Paiva, G. Berck, What is wrong with the Hausdorff measure in Finsler spaces. Preprint, arXiv:math.DG/0408413

7. J.C. Álvarez Paiva, E. Fernandes, Crofton formulas in projective Finsler spaces. Electron. Res. Announc. Amer. Math. Soc. 4 (1998), 91-100. 
8. J.C Álvarez Paiva, E. Fernandes, Fourier transforms and the HolmesThompson volume of Finsler manifolds. Int. Math. Res. Not. 19 (1999), 1031-1042.

9. J.C Álvarez Paiva, E. Fernandes, What is a Crofton formula? Math. Notae $42(2003 / 04), 95-108$.

10. J.C. Álvarez Paiva, E. Fernandes, Gelfand transforms and Crofton formulas. Selecta Math. (to appear).

11. J.C. Álvarez Paiva, I.M. Gelfand, M.M. Smirnov, Crofton densities, symplectic geometry, and Hilbert's fourth problem. In: "Arnold-Gelfand Mathematical Seminars, Geometry and Singularity Theory" (V.I. Arnold, I.M. Gelfand, V.S. Retakh, eds.), pp. 77-92, Birkhäuser, Boston 1997.

12. J.C. Álvarez Paiva, A.C. Thompson, Volumes on normed and Finsler spaces. In: "A Sampler of Riemann-Finsler Geometry" (D. Bao, R.L. Bryant, S.-S. Chern, Z. Shen, eds.), pp. 1-48, MSRI Publ., vol. 50, Cambridge University Press 2004.

13. R.V. Ambartzumian, A note on pseudo-metrics in the plane. Z. Wahrscheinlichkeitsth. verw. Geb. 37 (1976), 145-155.

14. R.V. Ambartzumian, V.K. Oganian, Parametric versions of Hilbert's fourth problem. Israel J. Math. 103 (1998), 41-65.

15. G. Bellettini, M. Paolini, S. Venturini, Some results on surface measures in calculus of variations. Ann. Mat. Pura Appl. (4) 170 (1996), 329 - 357.

16. W. Blaschke, Über die Figuratrix in der Variationsrechnung. Arch. Math. Phys., III. Reihe, 20 (1913), 45-61.

17. E.D. Bolker, A class of convex bodies. Trans. Amer. Math. Soc. 145 (1969), 323-346.

18. H. Busemann, The isoperimetric problem for Minkowski area. Amer. J. Math. 71 (1949), 743-762.

19. H. Busemann, Areas in affine spaces III. The integral geometry of affine area. Rend. Circ. Mat. Palermo (2) 9 (1960), 226-242.

20. H. Busemann, Geometries in which the planes minimize area. Ann. Mat. Pura Appl. (4) 55 (1961), 171-190.

21. H. Busemann, Problem IV: Desarguesian Spaces. Proc. A.M.S. Symp. Pure Math. 28 (1976), 131-141.

22. G.D. Chakerian, Integral geometry in Minkowski spaces. In: "Finsler Geometry" (D. Bao et al., eds.), pp. 43-50; Contempory Math. 196, Amer. Math. Soc., Providence, RI 1996.

23. G.D. Chakerian, D. Logothetti, Cube slices, pictorial triangles, and probability. Math. Mag. 64 (1991), 219-241.

24. M.M. Deza, M. Laurent, Geometry of Cuts and Metrics. Springer, Berlin 1997.

25. A. El-Ekhtiar, Integral geometry in Minkowski space. Ph.D. Thesis, University of California, Davis 1992.

26. E. Fernandes, Double fibrations: a modern approach to integral geometry and Crofton formulas in projective Finsler spaces. Ph.D. Thesis, Louvain-laNeuve 2002.

27. P. Funk, Über eine geometrische Anwendung der Abelschen Integralgleich- 
ung. Math. Ann. 77 (1916), 129-135.

28. R.J. Gardner, Geometric Tomography. Encyclopedia of Math. and Its Applications, vol. 58, Cambridge University Press, Cambridge 1995.

29. I.M. Gelfand, M.M. Smirnov, Lagrangians satisfying Crofton formulas, Radon transforms, and nonlocal differentials. Adv. Math. 109 (1994), 188227.

30. P. Goodey, W. Weil, Translative integral formulae for convex bodies. Aequationes Math. 34 (1987), 64-77.

31. P. Goodey, W. Weil, Zonoids and generalizations. In: "Handbook of Convex Geometry", vol. B (P.M. Gruber, J.M. Wills, eds.), pp. 1297-1326. NorthHolland, Amsterdam 1993.

32. H. Groemer, Geometric Applications of Fourier Series and Spherical Harmonics. Encyclopedia of Math. and Its Applications, vol. 61, Cambridge University Press, Cambridge 1996.

33. G. Hamel, Über die Geometrien, in denen die Geraden die Kürzesten sind. Math. Ann. 57 (1903), 231-264.

34. J.B. Kelly, Hypermetric spaces. In: "The Geometry of Metric and Linear Spaces" (L.M. Kelly, ed.), pp. 19-31. Lecture Notes in Math. 490, Springer, Berlin 1977.

35. G. Matheron, Random Sets and Integral Geometry. Wiley, New York 1975.

36. A.V. Pogorelov, Hilbert's Fourth Problem. Scripta Series in Mathematics. V.H. Winston \& Sons, Washington, D.C.; A Halstead Press Book, John Wiley \& Sons, New York 1979 (Russian Original: Izdat. "Nauka", Moscow 1974).

37. L.A. Santaló, Integral Geometry and Geometric Probability. Encyclopedia of Math. and Its Applications, vol. 1, Addison-Wesley, Reading, MA 1976.

38. R. Schneider, Zu einem Problem von Shephard über die Projektionen konvexer Körper. Math. Z. 191 (1967), 71-82.

39. R. Schneider, Über eine Integralgleichung in der Theorie der konvexen Körper. Math. Nachr. 44 (1970), 55-75.

40. R. Schneider, Convex Bodies: the Brunn-Minkowski Theory. Encyclopedia of Math. and Its Applications, vol. 44, Cambridge University Press, Cambridge 1993.

41. R. Schneider, On areas and integral geometry in Minkowski spaces. Beiträge Algebra Geom. 38 (1997), 73-86.

42. R. Schneider, On the Busemann area in Minkowski spaces. Beiträge Algebra Geom. 42 (2001), 263-273.

43. R. Schneider, Crofton formulas in hypermetric projective Finsler spaces. Arch. Math. 77 (2001), 85-97.

44. R. Schneider, On integral geometry in projective Finsler spaces. Izv. Nats. Akad. Nauk Armenii Mat. 37 (2002), 34-51.

45. R. Schneider, Crofton measures in polytopal Hilbert geometries. (in preparation).

46. R. Schneider, W. Weil, Zonoids and related topics. In: "Convexity and Its Applications" (P.M. Gruber, J.M. Wills, eds.), pp. 296-317, Birkhäuser, Basel 1983. 
47. R. Schneider, W. Weil, Stochastische Geometrie. Teubner, Stuttgart 2000.

48. R. Schneider, J.A. Wieacker, Integral geometry in Minkowski spaces. Adv. Math. 129 (1997), 222-260.

49. Z.I. Szabó, Hilbert's Fourth Problem, I. Adv. Math. 59 (1986), 185-301.

50. A.C. Thompson, Minkowski Geometry. Encyclopedia of Math. and Its Applications, vol. 63, Cambridge University Press, Cambridge 1996.

51. W. Weil, Kontinuierliche Linearkombination von Strecken. Math. Z. 148 (1976), 71-84.

52. W. Weil, Centrally symmetric convex bodies and distributions, II. Israel $J$. Math. 32 (1979), 173-182. 